\title{
SENTIDOS DE LO EXPERIMENTAL EN LA ETNOGRAFÍA CONTEMPORÁNEA. Un debate epistemológico
}

\author{
Eduardo ÁLVAREZ PEDROSIAN \\ Laboratorio Transdisciplinario de Etnografía Experimental, FIC-Udelar, Montevideo (Uruguay) \\ eduardo.alvarez@fic.edu.uy
}

\begin{abstract}
SENSES OF THE EXPERIMENTAL IN CONTEMPORARY ETHNOGRAPHY. An epistemological debate
\end{abstract}

Resumen: En este ensayo nos proponemos problematizar la concepción de lo "experimental" en la etnografía contemporánea a partir de preguntarnos sobre los sentidos que pueden considerarse al respecto, en particular aquellos que evaluamos como los más fértiles para su desarrollo. Un primer haz de sentidos nos exige plantearnos el rol de la experiencia en la producción de conocimiento etnográfico en todas las etapas del proceso. En segundo lugar, llegamos al corazón de la problemática epistemológica recuperando los sentidos de lo experimental provenientes de las ciencias naturales, las artes y la filosofía más afines a la labor etnográfica. Concluimos por último, con una serie de consideraciones sobre la necesidad de superar la dicotomía entre tradición e innovación de una forma pos-vanguardista, encontrando en la revolución permanente la mejor dinámica que se ajusta a esta estrategia de investigación e intervención.

Abstract: In this essay we propossal a problematization the concept of the "experimental" in the contemporary ethnography. We propouse questioning the senses which can be considerers about them, in particular those we estimate like a more fertile for his development. A first line of senses, it requires us to expose the role of experience on ethnographic knowledge during all steps of process. In a second time, we arrived to heart of epistemological problematic taking the senses of experimental from the natural sciences, arts and philosophy closer to the ethnographic labour. Finally, we concluse whith a series of consideration about the superation of dicotomy beetween tradition and innovation in a post-vanguardian way, finding in the permanent revolution the macht better dinamic for this strategic of research and intervention.

Palabras clave: Etnografía experimental. Producción de conocimiento. Creación conceptual. Laboratorio antropológico. Revolución permanente

Experimental Ethnography. Knowledge Production. Conceptual Creation. Anthropological Laboratory. Permanent Revolution 


\section{Horizontes contemporáneos de experimentación}

En este artículo nos proponemos una indagación epistemológica sobre los sentidos de lo experimental en la etnografía contemporánea. Nuestro problema se centra en la forma de concebir dicho carácter experimental, preguntándonos sobre las potencialidades y alcances que ello conlleva. Si bien podemos encontrarnos con una variedad de planteos, consideramos específicamente aquellos que a nuestro entender enriquecen el ejercicio de la etnografía habilitando líneas de desarrollo para la creación de conocimiento e intervención. Esperamos no caer en la tentación de ofrecer otra versión más de lo que sería una corta pero intensa historia comenzada míticamente en el famoso seminario de Santa Fe (Nuevo México) de 1984, del que se desprenden los llamados "posmodernos" (Clifford y Marcus, 1991). Si nos parece que plantearnos los sentidos de lo experimental puede ser útil y necesario, lo hacemos desde un punto de vista particular sobre la problemática, el cual va siendo explicitado a lo largo del ensayo.

El horizonte contemporáneo de ejercicio de la etnografía experimental que identificamos como potencial y merecedor de desarrollo, es hijo de esa deriva de tres décadas de inestabilidades, un "momento experimental" (Marcus y Fischer, 2000) que parece haber llegado para quedarse en las ciencias humanas y sociales, con especial foco en la etnografía, junto a su gran expansión. Se trata de la puesta en crisis de la representación, de la episteme moderna, en los diversos ámbitos de investigación cada vez más interconectados. Desde nuestro punto de vista, existe un trayecto posible, más o menos presente en investigaciones contemporáneas, donde se ha ido pasando "de la retórica a la producción de subjetividad" (Álvarez Pedrosian, 2011b). De todas maneras, esta etnografía, muy afín o directamente identificada a veces como post-estructuralista o perspectivista (Viveiros de Castro, Descola, Latour), es atacada desde aquellas posiciones que cuestionaron a la posmodernidad en su momento, ya que consideran que se trata de una nueva faceta de esta pero de menor radicalidad (Reynoso, 2015). El supuesto "agotamiento" de la etnografía experimental proclamado por este tipo de cuestionamientos no ha sido tal, más bien todo lo contrario. Reynoso (1991) planteaba que las producciones más significativas se habían desarrollado entre 1977 y 1982. Sus declaraciones de entonces se ubican incluso más cerca de lo que sería el comienzo del proceso que de nuestros días, y fue gracias a él, paradojalmente, que los llamados posmodernos de la antropología norteamericana se hicieron conocer en el amplio público castellanoparlante.

Desde nuestra experiencia de formación en el campo, a mediados de la década de 1990 del siglo pasado, en el contexto montevideano, la posmodernidad lo podía casi todo. Incluso los arqueólogos eran tocados por el "giro hermenéutico". Siempre desustancializar, sin parar, al punto de desviarse de cualquier construcción. Era una suerte de meta-teoría anti-teórica; en definitva una gran negación. No tardaron las cosas en cambiar, mientras el planeta se interconectaba, por lo menos en lo concerniente a las comunidades académicas donde la etnografía era practicada, estudiada y venerada. En el medio de las "antropologías del mundo" (Lins Ribeiro y Escobar, 2008), no parece haber espacio-tiempo para ese tipo de narcisismos, aunque siguen vigentes. Lo cierto es que la conectividad virtual, la migración de los conocimientos al ciberespacio y la multiplicación de escenarios de encuentro e intercambio, es decir, el desarrollo de la comunicación científica, ha aumentado exponencialmente la posibilidad de que nuevas expresiones sean identificadas. Entre ellas, se encuentra la que aquí tratamos.

En el planteo emblemático sobre la etnografía experimental, Marcus y Fischer (2000) llevan a cabo una suerte de cotrapunto epistemológico con la obra de Kuhn, o más exactamente, con la popularización académica de sus primeros trabajos en torno al concepto de paradigma. Como hemos expresado en otras oportunidades (Álvarez Pedrosian, 2008), en su momento esto parece haber sido realizado en una forma genérica, sin entrar en detalle. 
Existe una suerte de ambivalencia en el tratamiento del esquema de funcionamiento de las ciencias, donde Kuhn establecía dos momentos o dinámicas diferenciales: la ciencia normal y la extraordinaria. Los autores que sistematizan la cuetión de lo experimental a mediado de los años 80' del siglo pasado, se sostienen en la idea de excepcionalidad de la práctica científica sin paradigmas hegemónicos, donde la hibridación, yuxtaposición y electicismo de tendencias teóricas hacen al horizonte más o menos compartido a pesar de las grandes discrepancias.

Creemos que en estas décadas se ha puesto de manifiesto la condición de "emergencia permanente" del conocimiento en ciencias humanas y sociales, donde la etnografía ha cumplido un rol central, desde la experiencia del extrañamiento dentro de la cual se conjungan las perspectivas comprensivas y críticas sin necesidad de oponerse. En ello convergen tendencias como las sostenidas en la reflexividad de tipo crítico con las fenomenológico-hermenéuticas. Estos planteos tempranos, argumentan acertamedente a nuestro parecer acerca de la relación íntima de los planteos experimentales con obras clásicas de otros períodos de la antropología y la etnografía, en especial los trabajos del modernismo francés de las décadas de 1920 y 1930 previas a lo que se conoció como estructuralismo (Clifford, 1995), e incluso reconocen en trabajos como los de Bateson y Mead elementos innovadores al respecto (Marcus y Fischer, 2000). En todo caso, la dimensión mediacional, comunicacional de la etnografía y su producció de conocimiento es decisiva. Si bien en la primer década se hizo hincapié en "la mesa" (Velasco y Díaz de Rada, 1997), especialmente en las estrategias escriturales, y más en general representacionales, puestas en crisis, les siguió con el cambio de milenio el énfasis en los dilemas de lo experimental en "el campo" (Estalella y Sánchez Criado, 2016). El paso del modelo o metáfora del "texto" al del "diálogo", es aplicable a ambas instancias de la producción de conocimiento (Marcus y Fischer, 2000: 59):

"Así, si bien la mayor parte de la experimentación no implica una ruptura tajante con la práctica etnográfica del pasado, constituye sin embargo una reorientación fundamental. Las etnografías siempre han sido en cierto sentido experimentales [...] En un período experimental, el peligro es precisamente que se lo clausure antes de tiempo, que algunos experimentos se tomen equivocadamente como modelos, den lugar a una corriente mecánica de imitadores o restablezcan convenciones sobre bases débiles. Determinados experimentos se plantean problemas particulares a fin de examinarlos, cosa que hacen más o menos bien; pueden llevar al límite determinada cuestión, y su contribución está en demostrar ese límite. Una obra en particular puede cumplir una tarea que no tendría objeto repetir. Pero una línea de experimentación puede perder su razón de ser si se vuelve identificable como subgénero" (Marcus y Fischer, 2000: 75-76).

\section{La fuerza de las prácticas en el quehacer etnográfico}

Vayamos a continuación hacia una primera dirección de los sentidos de lo experimental en la etnografía contemporánea. Para ello debemos descender de lo experimental a la experiencia más en general. En otras oportunidades (Álvarez Pedrosian, 2011, 2014a) hemos tomado la tesis de Sáez Rueda (2002) acerca del devenir de las dos tradiciones filosóficas occidentales, las conocidas como continental y analítica, para ubicar a la etnografía contemporánea en el mapa de las estrategias cognoscentes. Desde la primera vertiente, según su planteo, podemos encontrar una trayectoria que va de Husserl y pasa por Heidegger, donde la preocupación del pensamiento es la de alcanzar una "mundanización del sentido", mientras que en la segunda tradición, vamos de Frege a Wittgenstein, buscando una "na- 
turalización del significado" (Sáez Rueda, 2002). Ciertamente, el "hiato otnológico" entre ambas tradiciones sigue siendo fuerte, pero este horizonte pone en evidencia una preocupación semejante por concebir al pensamiento, y el con él a la teoría, como una forma de práctica. Efectivamente, la "práctica teórica" es una "práctica entre las prácticas" (Álvarez Pedrosian, 2005), lo que equivale a decir que es una meta-práctica al mismo tiempo que una práctica intersticial entre las demás, lo que es muy diferente de concebirla como una entidad trascendente. La factualidad de la teoría, es algo que la etnografía fue procesando a lo largo de su historia, para nada linea ni evolutiva. En tal sentido, el "momento Writing Culture" (Clifford y Marcus, 1991; Marcus, 2008) puede ser visto como un efecto superficial de un movimiento epistemológico y ontológico mucho más profundo y de muy larga duración en términos temporales.

En una etnografía experimental, por tanto, la experiencia de creación de conocimiento, desde "el campo" a "la mesa" (Velasco y Díaz de Rada, 1997) y viceversa, es lo que constituye su plano de inmanencia. No tener presente la problemática de la experiencia, para nada cerrada, es anular de antemano la posiblidad misma de toda experimentación. Y ello tanto en un sentido más asociado al dogmatismo empirista, donde investigar es verificar o falsear lo que la teoría previa informa, como en otro sentido opuesto, donde parece que dichas herramientas conceptuales fruto de múltiples experiencias previas no tengan nada que hacer en el acontecimiento de investigar. No es aquí momento de reiterar las consideraciones realizadas a propósito del pragmatismo que creemos anidó en la etnografía fundacional y que fuera emergiendo y siendo utilizado como fuente más o menos explícita de alimentación a partir de la denominada "crisis de la representación" (Álvarez Pedrosian, 2011b, 2014a). De nuestro análisis tan solo rescatemos en esta ocasión el carácter "intempestivo", al mejor estilo nietzscheano, de la experiencia etnográfica; lo que en los términos del primer pragmatismo también aparece como condición básica de la experiencia en tanto conmoción o perturbación a cada paso, "aparición de la diferencia" y "alteración” según el propio James (2009: 177). Estar abierto a la afectación (Favret-Saada, 2005), ser atravesado por los procesos gracias al involucramiento más o menos colaborativo en medio de los flujos de procesos en cuestión, es consustancial a la propuesta. Qué hacer con todo ello, cómo producir conocimiento reflexivo que si bien comience con ello pueda ir más allá de un "género confesional" amarrado a un "yo testifical" (Geertz, 1989; Marcus y Fischer, 2000; Reynoso, 1991), es algo que si bien estuvo presente desde el método estandar fundacional se vuelve mucho más importante una vez se asume la conmoción de la condición mediacional y transitoria de toda producción de conocimiento sustentada en la etnografía, encontrando en ello una virtud más que un defecto (Marcus, 2008).

Ser más precisos y rigurosos con la forma de tratar con las experiencias, como veremos hacia el final de este artículo, constituye un camino de maduración de lo experimental. Pero un primer paso es este ponerse en sintonía con la naturaleza práctica de la existencia, incluida la producción de conocimiento. Y si bien, en otras esferas de actuación esto puede ser minimizado por protocolos y programaciones que intenten dominar lo más posible el azar, lo intempestivo y múltiple de la proliferación experiencial, pues es necesario para solucionar cuestiones instrumentales, en la exploración de los fenómenos humanos con la finalidad de la creación de conocimiento y pensamiento, así como de una aprehensión sensible y afectiva de dichas experiencias, más bien se trata de todo lo contrario. Dejarse llevar, entonces, por lo que las propias experiencias tienen para enseñarnos, es el primer haz de sentidos de lo experimental en la etnografía contemporánea. La cuestión de cómo llevar esto a cabo, nos remite a lo mejor de la tradición etnográfica en sus diversas vertientes histórico-regionales. Y ello, reiteramos, implica tanto al proceso de análisis y síntesis del material generado, o sea, a "la mesa" (Velasco y Díaz de Rada, 1997), como al trabajo de campo. De las discusiones heredadas del llamado posmodernismo todavía se insiste desde ciertas posiciones en que esta deriva conlleva un "esteticismo" carente de politicidad. Por el contrario, no 
hay nada más político que esta asunción y compromiso con la experiencia, en un realismo sofisticado donde todo tipo de acciones son parte de la dimensión de relaciones de fuerza.

Sabemos lo que parece ser el talón de Aquiles de todo ello en los términos de la teoría del conocimiento: lo precario de la generalización alcanzada por un conocimiento producido de tal forma. Pero ello ya ha sido debatido, fundamentado y puesto en marcha en diferentes programas de investigación a partir de la sociología compresiva de Weber y sus descendencias. Más recientemente en el tiempo, y recordémoslo, sin querer volver a escribir una historia de lo experimental en la etnografía de estas últimas décadas, el interpretativismo de Geertz (1996) se sostuve en esta tradición de pensamiento alemán sobre la subjetividad y lo que Dilthey llamó las "ciencias del espíritu”. La generalización se da a partir de la profundización en las particularidades de cada caso, siempre en articulaciones múltiples con otros, en redes con sus densidades relativas. Generalización por tanto existe; no es que nos perdemos en una suerte de fragmento aislado de experiencia humana. Como se reconoce que la condición general es esta singularización, sostenida en lo irrepetible de cada acontecimiento, el problema gnoseológico no es tal.

Dentro del espíritu ecléctico de la etnografía, afirmado cada vez más durante las últimas décadas en cuestión, otros abordajes teóricos-metodológicos donde la generalización se da en forma horizontal más que vertical, extensiva más que intensiva, también pueden combinarse con la forma más asociada a lo cualitativo de la exploración de los sentidos en lo experimental. Cuadros y diagramas, todo tipo de formalización más o menos funcional proveniente de la tradición cientificista, son componentes indispensables de la experimentación, nuevamente, tanto en su ejercitación por parte del etnógrafo y otros sujetos involucrados en la pesquisa, como en la forma de procesar lo que ello nos brinde. Lo que sí debemos reconocer, desde nuestro punto de vista, es la preminencia de la cualidad frente a la cantidad sea esta la que sea, pues esta última sólo cobra valor a partir de lo que tiene para informarnos en relación a la primera. La etnografía como producto, es concebida como una cartografía, en el sentido en que Deleuze y Guattari (1997b) lo postulan como principio rizomático. Esta etnografía cartográfica posee componentes variados. En otras ocasiones los hemos identificado como provenientes de las tradiciones científica, filosófica y artística respectivamente: los planos de coordenadas con sus funciones y la abstracción de los cuadros y sus referencias, la problematización conceptual que hace de lo anterior algo relativo y transitorio a la vez que lo proyecta o le da profundidad, y la aprehensión estética que permite captar, transmitir y generar nuevas experiencias sensibles (Álvarez Pedrosian, 2011b, 2014b).

\section{A la búsqueda de la bifurcación insospechada}

Un segundo haz de sentidos que consideramos está en juego en la caracterización de la etnografía experimental, o del ejercicio experimental de la etnografía, hace referencia a las direcciones que se buscan promover explícitamente en las investigación de este tipo. Junto al ponerse en sintonía en las experiencias de producción de conocimiento en todas sus instancias, siendo por demás sensible y percibiendo cada alteración, aparición de componentes de variada índole desde todas las subjetividades y entidades involucradas como oportunidades a ser exploradas, el etnógrafo experimental busca reflexivamente, e incluso provoca en forma deliberada alteraciones en los procesos que permitan abrir nuevas sendas. En este aspecto es quizás donde mejor se pone en discusión la diferencia entre lo experimental hererado de las ciencias naturales y de las artes, y la cercanía de la antropología etnográfica a estas últimas. Al respecto existen buenos e interesantes antecedentes en la teoría del pensamiento y el conocimiento, así como en la epistemología profesional, encontrándonos en la llamada nueva filosofía de la ciencia con debates muy intensos desde unas décadas previas a las correspondientes a la aparición de estos debates en el campo de la antropología y demás ciencias humanas y sociales donde la etnografía fue ganando terreno. 
En este segundo tipo de sentidos de lo experimental, la propuesta de Feyerabend (1994) es la que más se acerca al quehacer etnográfico. Incluso promovió los puentes entre las ciencias naturales y las artes, apostando por un cambio radical de concepción, con ejemplos tomados de la historia de las ciencias, donde el dogmatismo defendido con virulencia por aparentes progresistas como Kuhn es dinamitado. La repetición como base del método, la anulación de la imaginación y la creatividad, se condice con una ontología donde los elementos que pueblan el universo siguen siendo aquellos átomos primarios cartesianos. Sosteniéndose en el empirismo de Mill, Feyerabend recurre a la noción de "proliferación" como fundamental para la comprensión de la naturaleza estudiada, sea esta humana o no-humana, incluyendo la naturaleza de la investigación científica sobre y dentro de ellas.

Uno de los puntos centrales en este debate es el papel que ocupar el error. La vieja teoría del ensayo-y-error, retomada y alzada como base de su propuesta por parte de Popper, si bien reintegra la crítica en el corazón de la empresa científica ante las simplificaciones lógicas del verificacionismo, mantiene una perspectiva lineal del proceso. Pero bien, el aprender de los errores es sustancial, incluso buscar su generación para poder aproximarse a lo que sea lo verosímil en la construcción de nuestras explicaciones. Recordemos que en el planteo kuhneano no hay lugar para el error, pues su holismo relativista es de un tipo dogmático, lo que lleva a una suerte de disolución entre lo que se cree conocer y lo que se conoce efectivamente (para no confundir con falsos problemas derivados de oposiciones mal planteadas entre lo teórico y lo práctico, entre los contextos de justificación y descubrimiento). Feyerabend retoma el falsacionismo intentando complejizarlo, aceptando las cadenas de ensayos-y-errores pero abriéndolas como una red a múltiples direcciones, en tanto las "reglas" tarde o temprano son "infrigidas", y deben serlo para estimular el desarrollo del conocimiento científico (Feyerabend, 1994: 14).

Cuando se trabaja a partir de los sentidos de lo experimental aquí propuestos en primer término, presente tanto en la concepción de lo acontecimental nietzscheano como en lo experiencial jamesiano, los errores no son algo tan fácil de identificar. Pero esto no quiere decir que la alternativa sea ir hacia delante ciegamente. Esta problemática encuentra en el racionalismo aplicado de Bachelard y la reflexividad cultivada por Bourdieu una salida productiva ante lo que consideramos puede ser un callejón sin salida. Se trata en definitiva de aprender sobre la marcha, una marcha llena de tropiezos, de "obstáculos epistemológicos" más que de errores, en los sentidos tradicionales y comunes en que se planteó en nuestra cultura. Los obstáculos nos interpelan profundamente, son consustanciales al propio conocimiento, nos obligan a innovar, a ensayar, a experimentar, buscando posibles caminos para su superación. Son siempre "confusos" y "polimorfos", no se superan de una vez sin más (Bachelard, 2004).

Precisemos más este conjunto de sentidos de lo experimental, y su relación con las ciencias y las artes, pues es aquí donde se concentra lo más denso del debate, o donde creemos que debe hacerse el foco para avanzar en la dilucidación de la problemática epistemológica más o menos explícita en la labor etnográfica contemporánea. El sentido tradicional de lo experimental, proveniente de las llamadas ciencias experimentales, tiene al laboratorio como espacio-tiempo primordial. Se trata de una disposición integral de agentes de variada índole donde se intenta matener el mayor control posible sobre las variables en cuestión, de forma de poder identificar con la mayor precisión y discernimiento posible los componentes específicos que se pretende conocer. De esta forma se diferencia entre variables y constantes, se establecen las llamadas "condiciones iniciales", así como los resultados obtenidos. El proceso es cuidadosamente diseccionado en etapas, donde las hipótesis son contrastadas con los hechos.

Gracias a las etnografías de laboratorio, consideradas aquí como experimentales, se llega a la constatación de que las perspectivas verificacionista y falsacionista no dejan de ser actos de construcción (Latour y Woolgar, 1995). Esto no invalida para nada la producción de 
conocimiento en tales circunstancias, si se comprende la implicación entre los componentes puestos en juego en el acontecimiento del experimento. Si bien es cierto que en un primer momento, estos trabajos alimentaron una visión retórica y en especial "textualista" de la investigación al estilo de la antropología autocalificada de posmoderna (Bourdieu, 2003), desde el pensamiento complejo generado por filósofos y científicos naturales se subraya este constructivismo sin caer en tales simplificaciones. Ya desde principios de siglo XX con la termodinámica, la física cuántica y la relatividad, se pone en jaque la actividad de dominio, pero no por ello desaparece lo experimental. Lo que ocurre es que sufre una importate mutación:

"El diálogo experimental con la naturaleza, que la ciencia moderna se descubre capaz de llevar a cabo sistemáticamente, no supone una observación pasiva, sino una práctica. [...] El paso experimental constituye entonces un arte, es decir, que reposa sobre una habilidad y no sobre reglas generales y se encuentra por ello sin garantía, expuesto a la trivialidad y a la ceguera; ningún método puede eliminar el riesgo de perseverar, por ejemplo, en una interrogación sin pertinencia. Arte de elección, de discernimiento progresivo, de examen exhaustivo de todas las posibilidades de respuesta de la naturaleza en una situación precisa, el arte experimental consiste en escoger un problema para formular una hipótesis teórica y en reconocer en la complejidad proliferante de la naturaleza un fenómeno susceptible de encarnar las consecuencias de este decreto general [...] Ciertamente, como subrayan los críticos, cualquiera que sea la respuesta, sí o no, la naturaleza se ve siempre forzada a confirmar el lenguaje teórico en el cual se le dirije la palabra. Pero este mismo lenguaje evoluciona según una compleja historia en donde intervienen a la vez el balance de las respuestas obtenidas de la naturaleza, la relación con otros lenguajes teóricos y también la exigencia que renace sin cesar bajo nuevas formas, nuevas preguntas, de comprender la naturaleza según lo que cada época define como pertinente. [...] El protocolo de diálogo experimental representa para nosotros una adquisición irreversible" (Prigogine y Stengers, 1990: 67 a 70).

A pesar de querer abrirse a un nuevo diálogo con la naturaleza, la ciencia natural requiere de esa puesta en suspenso o proyección de "plano de coordenadas" que busca referenciar los fenómenos (Deleuze y Guattari, 1997a: 203). Gracias a ello es posible el discernimiento y la formalización, como principal actividad cognoscente, lo que asegura a su vez la comunicabilidad de la experiencia, incluso su reproductividad por parte de otros en otros tiempos y espacios. Como es sabido, esto es casi imposible en la mayoría de las dimensiones de trabajo de las ciencias humanas y sociales, pues la naturaleza abordada en este sentido es la misma en la que está inserto el investigador, y por tal motivo la separación entre objeto y sujeto es de otra índole. Pero algo de científicifidad persiste, por lo fértil que resulta. En tal sentido, donde ya la ciencia se reconoce íntimamente conectada con el arte, pero diferente a él, la etnografía encuentra los insumos necesarios para llevar a cabo esa misma operación de discernimiento, o sea, de identificación de los elementos, convertidos en variables y constantes, eso sí, en forma circunstancial y siempre desde la reflexividad que implica tener presente la operación misma de su construcción.

Desde las artes, las vanguardias estéticas contemporáneas a estos cambios de principios de siglo XX hicieron del experimento una suerte de leitmotiv. El dadaísmo y el surrealismo en concreto, son de extrema importancia para el desarrollo de la etnografía experimental (Clifford, 1995), como el cine documental soviético revolucionario (Russell, 1999). Podemos rastrear esta práctica en pensadores como Benjamin, tal como el propio Taussig (1995) lo reconoce enfatizando la utilización del "montaje" de tipo constructivista (Álvarez 
Pedrosian, 1999), o en movimientos como el situacionismo de los años sesenta y sus exploraciones urbanas en forma de "derivas" tan influyente hasta nuestros días (Careri, 2009).

"El término "experimental" se difunde a partir de los años cincuenta, en particular en relación con la música. [...] aparece como el uso de nuevas tecnologías de producción, grabación y reproducción. [...] como una forma de liberación del sonido respecto a las intenciones del músico, de tal modo que la "música", se hace a sí misma como John Cage diría, "indeterminada", como resultado del azar. [...] Para Cage, una acción experimental sería aquella de la cual no se pueden prevenir sus resultados, una acción necesariamente única" (Sansi, 2016: 67-68).

Desde el arte, lo experimental se concibe en los sentidos más radicales de juego, manipulación, incluso de improvisación, donde se lleva al extremo la posibilidad de comenzar algo nuevo, sui generis, a pesar de saberse que esto es imposible. Como bien señala Sansi (2016) al respecto, este sentido parece diametralmente opuesto al científico antes planteado. Para la etnografía, constituye la estrategia de aprehensión de los fenómenos (Álvarez Pedrosian, 2011b, 2014a), tanto en lo concerniente a los afectos presentes en las experiencias del trabajo de campo como en las del procesamiento y análisis de la información, dinámicas entre "el campo" y "la mesa" (Velasco y Díaz de Rada, 1997) que son parte de la misma práctica singular de etnografiar tal o cual fenómeno en circunstancias concretas, solo posibles de ser reproducidas (en el sentido científico antes planteado) en dimensiones macro y a escalas de gran generalización, algo también importante como ingrediente de un abordaje etnográfico.

Un tercer componente, indispensable según nuestra perspectiva, es el proveniente de la filosofía, en tanto arte de "crear conceptos" (Deleuze y Guattari, 1997a, 11). Al igual que las operaciones provenientes de las ciencias y las artes, se conjuga en la etnografía, en especial en aquella que aquí nos interesa (Álvarez Pedrosian, 2011b). Quizás es en esta dimensión o vertiente cognoscente donde lo experimental ha sido menos abordado, sustentado y defendido, salvo en aquellas tendencias filosóficas más afines con las artes, dando lugar a "planos complejos difíciles de calificar" como reconocen los mismos Deleuze y Guattari (1997a, 219). Y es que como hemos afirmado también en varias oportunidades, esta composición heteróclita de la etnografía como estrategia de abordaje y construcción de conocimiento no pretende diluir las diferencias entre arte, ciencia y filosofía, o si se quiere, ese no es nuestro argumento. La etnografía fue constituyéndose de tal forma, que las operaciones cognoscentes del discernimiento científico, la aprehensión estética y el sobrevuelo de los conceptos filosóficos, se ensamblan conformando un tipo de saber con sus particularidades fruto de esta síntesis parcial (Álvarez Pedrosian, 2011b, 2014b). La etnografía es mucho más que un simple relevamiento de ejemplos concretos subsumidos a conceptos abstractos, donadora de materia prima para el filosofar: es "un modo de permanecer conectados con procesos sociales abiertos e incluso misteriosos y con incertidumbres" (Biehl, 2016: 245). Se trata de una estrategia que al combinar la creación conceptual desde la experiencia del extrañamiento inmersa en los procesos dialógicos y polifónicos de producción de subjetividad (Álvarez Pedrosian, 2011b), es capaz de desarrollar un conocimiento diferente pero ligado íntimamente a este esfuerzo de conceptualización desde la "inversión del platonismo" (Álvarez Pedrosian, 2014a), superando la dicotomía esencia-apariencia o modelo-copia en pos de una apertura existencial radical, la de la fuerza de los simulacros más allá de lo representacional, más allá de la diferencia y lo Otro (Deleuze, 1989: 263).

Cuando se concibe al pensar como acontecimiento, la tarea filosófica también es una cuestión de experiencia (que no es lo mismo que vivencia), y la experimentación cobra un rol primordial cuando el azar y la intempestividad asechan al concepto, más que quedar eliminados gracias a una trascendentalización al estilo del "mundo de las ideas” platónico. 
Esta experimentación, en su componente conceptual o filosófico, se expresa con fuerza en el tipo de gnoseología elaborada por Vaz Ferreira (1957) en el Uruguay de principios del siglo XX, en diálogo con el pragmatismo de James y el vitalismo de Bergson, en una suerte de antecedente casi desconocido más allá de Iberoamérica. Dinámicas cognitivas con sus respectivas técnicas y métodos de trabajo, se orientan en esta dirección, donde la conciencia es un fluir, y las ideas son entidades vivas. El pensar como acontecimiento, en medio de las prácticas vitales, implica la identificación de un "psiqueo afectivo" que marca las trayectorias y devenires, a partir de la calibración o "graduación de la creencia", buscando los "fermentos pensantes" con los que se alimentan a su vez nuevos procesos creativos (Álvarez Pedrosian, 2009). En este sentido, por tanto, la etnografía es experimental en tanto cada investigación es una oportunidad para replantearse lo más posible las cosas, para poner en crisis los presupuestos de partida reflexivamente, para buscar otras líneas de interpretación, tensionando los límites de lo considerado como lo real y los argumentos sobre sus características o cualidades definitorias.

Por último, esta experimentación no es solo epistemológica, sino ontológica, y allí radica el pliegue entre la metodología etnográfica y su objetivo de investigación (o su objeto de estudio en términos clásicos), el que podemos identificar como el análisis de los procesos de subjetivación, en un laboratorio de experimentación que no es otro que la propia existencia de lo humano en devenir (Rabinow, 2009). Conlleva pensar el "diseño de ambientes para la vida" (Ingold, 2012), más allá de debates también existentes entre las diferentes vertientes antropológicas en cuestión y el rol de la etnografía en todo ello (Ingold 2008). Esto se realiza según el "pluralismo cognitivo" (Lins Ribeiro y Escobar, 2008) que ha caracterizado a las ciencias antropológicas por lo menos desde la gestación de la etnografía, a partir de "comunidades epistémicas" en entornos "para-etnográficos": "El laboratorio científico es el ejemplo paradigmático, pero creemos que el ethos experimental es parte de la estructura contemporánea y se manifiesta en incontables lugares..." (Holmes y Marcus, en Estalella y Sánchez Criado, 2016: 17).

Es, en tal sentido, de la articulación entre técnicas, métodos y teorías, que la etnografía experimental es coherente con sus intereses y finalidades, pues lo que se concibe como digno de ser estudiado e intervenido es una entidad abierta y múltiple, experimental. Lo que décadas atrás era identificado como las "diferencias experienciales a través de las fronteras culturales", donde era "central el enfoque en la persona, el yo y las emociones" (Marcus y Fischer, 2000: 110), se ha proyectado en todo el universo de creación y re-creación de formas de ser en tanto prácticas y haceres productores de subjetividad. El interés por trabajar en medio de las relaciones de fuerza, lo que en su momento constituyó el intento por recuperar la crítica política tras los juegos de formas retóricas, ha dado resultados, al mismo tiempo que la etnografía se proyecta en vastas zonas del volumen epistemológico de las ciencias humanas y sociales y más allá de este.

Sostener un trabajo experimental desde la etnografía contemporánea puede ser una militancia constante para la superación del largo enfrentamiento entre dogmáticos y escépticos primero, entre racionalistas y relativistas más acá en el tiempo, en una historia del pensamiento occidental de fuerte presencia global, en un contexto de hibridación, solapamientos y eclectisimos varios con otras fuentes de pensamiento más o menos en resistencia. A lo largo de estas últimas décadas, como hemos planteado en este ensayo, los combates encarnizados entre diferentes formas de uno y otro de los polos del binomio en las ciencias humanas y sociales y la filosofía cercana a estas, ha dado paso a proyectos interesantes desde nuestro punto de vista. Si bien para muchos, y en muchos sentidos justamente, lo experimental puede volver a caer en el polo del escepticismo y más cercanamente, del relativismo epistemológico e incluso ontológico, solo puede llevarse a cabo junto a una dosis igual de fundamental de las mejores virtudes del racionalismo. 
Similar a como Bachelard planteó su "racionalismo aplicado" como superación de las perspectivas del empirismo ingenuo y el racionalismo dogmático, es posible llevar a cabo la tarea investigativa desde un relativismo sofisticado, es decir, no peleado con lo real, lanzado por el contrario a la lucha por su conocimiento. Las duras críticas de Bourdieu a Latour y otros (Bourdieu, 2003: 51-61), en un momento donde aún los "estudios de laboratorio" eran la punta de lanza, parecen no haber tenido mucho eco posteriormente. Creemos importante incorporarlos al debate epistemológico contemporáneo, pero desde una perspectiva a su vez diferente. En síntesis: habitar los intersticios entre la filosofía y las ciencias humanas y sociales no solo es inevitable, sino necesario. De hecho, así lo venimos haciendo desde que las últimas se desprendieron de la primera, y pese a todo tipo de normas y prescripciones: las tentaciones no son solo cuestión de diversión e intensidad libidinal ante los abismos y peligros de lo inconcluso, sino una obligación ético-política.

¿Qué peso le damos a lo que existe a piori en nuestras experiencias y cómo se puede ir más allá de ello? El último Foucault (2002), al plantear una "ontología del presente o de nosotros mismos", retomaba el proyecto kantiano pero para invertirlo: lo universal es ahora el punto de partida, para alcanzar lo singular y singularizante, lo que constituye un "franqueamiento de los límites de lo posible". Y esto en los hechos que se investigan como desde la práctica de investigación que se involucra en estos, interveniendo en tal sentido. Ciertamente la reproducción de los establecido es de una fuerza contundente, y muchas veces se olvida ello en pos de esta búsqueda de las alternativas emanadas o emergidas del contexto de los fenómenos en cuestión. Pero incluso la repetición de lo que sea se lleva a cabo de formas variadas, lo que puede tener a su vez una lógica propia, como el estructuralismo lo planteara a mediados del siglo pasado. No se trata de un conjunto limitado de reglas de construcción, sino de múltiples, que sí son seleccionadas, a las que se remite para las operaciones de producción de lo real. Es por esta razón que el conocimiento debe aventurarse más allá de estos límites, relativizando lo real en pos del laboratorio donde habitan entidades de variada índole, muchas de las cuales no tienen casi probabilidades para darse un lugar entre lo existente (Álvarez Pedrosian, 2016). Llegar a ello, solo es posible si algo de todas formas logra asomar "rasgando el velo de las cosas familiares", para retomar una expresión de Malinowski (1989: 56) en su diario íntimo póstumamente publicado, y que tanto revuelo cauzara en toda esta historia desde la reformulación del pensamiento antropológico de la segunda mitad del siglo XX.

\section{Conclusiones: la madurez del trabajo experimental}

Como hemos planteado, si bien podemos ubicar la utilización primaria de los términos "etnografía experimental" en la antropología norteamericana autoproclamada como posmoderna allá por los comienzos de la década del 80’ del siglo pasado, la cuestión es mucho más vasta y compleja. Incluso se entrecruzan otras tendencias regionales y locales, en el contexto contemporáneo de las "antropologías del mundo" que se definen en un horizonte poscolonial o decolonial (Lins Ribeiro y Escobar, 2008). La experimentación también hace a este aspecto, a la existencia de otras tradiciones de pensamiento y conocimiento. Esto incluso se da más allá de la dicotomía norte-sur, en el entendido de que si bien siguen existiendo fuertes condiciones estructurales de dominación entre los nodos y redes de producción académica, las relaciones transverales e intersticiales subalternas desbordan incesantemente y en forma creciente los viejos esquemas occidentales de hegemonía y subalternidad.

La etnografía experimental, por tanto, es muchísimo más que lo representado por aquellos trabajos identificados en su momento por Reynoso (1991: 36-37), producidos en la órbita norteamericana en su gran mayoría, principalmente entre 1977 y 1982. Uno de sus argumentos de por entonces sigue siendo desde nuestro punto de vista por demás importante: "La idea misma de una etnografía experimental es contradictoria, porque implica 
continuidades, progresos y vanguardias típicos de la modernidad" (Reynoso, 1991: 38). Esa contradicción, creemos, puede ser superada, el pos de una lógica de la investigación para las ciencias humanas y sociales que pone en tela de juicio teorías epistemológicas más o menos establecidas aún, pero que nos obliga a ir más allá de la discución entre modernidad y posmodernidad. Los esfuerzos por articular la explicación comprensiva sobre el oficio interpretativo y reflexivo a un mismo tiempo han ido madurando paralelamente a esta otra historia, y en un contexto inter y trans-disciplinario inevitable de ser considerado para captar la magnitud del fenómeno epistemológico (Hastrup y Hervik, 1994; Rodríguez Victoriano, 2004; Atkinson, Delamont y Housley, 2007).

Se trata de abrir el juego, romper el conjunto cerrado, ver qué sucede en los límites. Este arriesgarse, donde el deseo sostiene la confianza en el proceso, es equipado por una caja de herramientas valorada para tales fines. Dichas herramientas genéricas son puestas en uso de forma singular. Poder hacer esto explícito, incluso buscar que suceda al tomarlo como regla, es lo que define lo experimental. No existe la inocencia absoluta: una versión de una creatividad extrema no es lo que define a la etnografía como estrategia de producción de conocimiento. Es una creatividad regulada, una apertura llevada a cabo de la forma más favorable posible para aprender de lo que allí acontece. Sí existe un salto al vacío, una "ruptura" con la doxa tanto interna como externa al propio campo, a pesar de todo lo a priori que existe y se lo reconoce como fundamental. Por ello se trata siempre de un "ensayo", tanto en los términos científicos naturales como artísticos, en especial literarios.

Nada de esto es significativo, para los directamente involucrados así como para otros agentes de otros contextos sociales, políticos y culturales, si no hay una "reconstrucción racional" del proceso, a posteriori, donde se pierda lo menos posible de lo valioso de la experiencia sucitada en "el campo", ahora en "la mesa" (Velasco y Díaz de Rada, 1997). La experimentación continúa, cada vez en forma más "transmediacional” (Walley, 2015). En esto es, quizás en lo que más se insistió en las décadas pasadas (Russell, 1999), pasándose ahora a pensar más en lo primero, en "el campo". De allí la proliferación de etnografías "colaborativas", como nuevo "imperativo" (Marcus, 2013). Esto es algo que en el contexto de las universidad latinoamericanas hermanadas por la Reforma de Córdoba de 1918 tenemos presente desde hace casi un siglo, y que en los últimos años venimos trabajando en la Universidad de la República como la "integralidad" entre la investigación, los procesos de enseñanza-aprendizaje y la extensión o actividades en el medio social, teniendo a la etnografía como una de sus protagonistas (Álvarez Pedrosian, 2011a).

La necesidad de perfeccionar o potenciar la calidad de la estrategia investigativa no es sinónimo de estandarización. Por el contrario, se trata de no caer en ello. La "revolución permanente", a partir de rupturas continuas como lo planteaba Bachelard (Bourdieu, 1999) constituye el ideal o la tendencia de una disposición para la creación de conocimiento de este tipo. No se trata de dialectizar la oposición, sino de envolver un elemento en el otro, y así intentar, con constancia, superar la dualidad: trabajar rigurosamente con creatividad y creativamente buscando el rigor. Por el grado de delicadeza o fragilidad de esta operación, en el umbral mismo de conversión de las fuerzas de poder en formas de saber, en la disputa sobre qué es lo real, la etnografía experimental que nos parece interesante desarrollar se encuentra necesariamente asediada. La experiencia del extrañamiento como soporte existencial del investigador, y los conocimientos producidos en nuevas experiencias derivadas de ello, desencadenan posteriormente nuevas realidades, más o menos materializadas, encarnadas en nuevas relaciones de fuerza y sentidos posibles (Álvarez Pedrosian, 2011b).

Por tal motivo, en un último sentido de lo experimental aquí enunciado, este tipo de estrategias cognoscentes se asumen desde la implicancia como práctica de desnaturalización y creación conceptual, durante y después de cada proceso de investigación-intervención. La experimentación sigue, de alguna manera, en los efectos que pueden irse identificando y las nuevas conexiones posibles de prácticas a partir y mediada por la labor y el producto etno- 
gráfico, en una pregunta ética que obliga a evaluar lo experimental antes, durante y después del proceso en la medida de nuestras posibilidades (Irwin, 2007), lo que afecta las vidas e incluso las muertes de los sujetos partícipes de las experiencias en cuestión (Biehl, 2016).

¿No son los llamados "clásicos", aquellas obras que lograron conmocionar su tiempo y/o el tiempo por venir? En una dinámica de "revolución permanente", no hace falta o es incluso perjudicial pretender la actitud vanguardista de la ruptura por la ruptura misma, lo mismo que pretender conservar los conocimientos previos a todo precio, como finalidad en sí. Y los llamados productos "clásicos" de la cultura, cada vez más mediatizados en los diversos territorios civilizatorios del planeta y sus flujos tranversales (entre ellos escritos y películas etnográficas emblemáticas) siguen vivos, en la medida que siguen siendo aprehendidos y utilizados, y con ello, generando efectos insospechados, nuevos sentidos para quienes habitan el presente respectivo.

Quizás lo que más interpela la etnografía experimental al resto de campos o espacios más o menos autonomizados de la producción de conocimiento en ciencias humanas y sociales contemporáneas, así como en otras áreas más aplicadas donde ha ido ganando terreno, sea esta forma de trabajo donde tradición e innovación se fusionan. Una crítica de la crítica, que procura generar porliferaciones y procesar bifurcaciones de forma aprehensible y con el mayor rigor posible para su incorporación, puede encontrarse con abundancia en el arte, en particula en la música. Lo que hizo Piazzolla con el tango es un claro ejemplo de ello. Interminables polémicas en su propio tiempo ante presuntos defensores de la mejor tradición rioplantese, que suscitó incluso "batallas campales" en conciertos, con sillas volando por los aires. Según sus propias reflexiones (Piazzolla en Montes-Baquer, 2007), la autenticidad de su trabajo no fue para él un fin en sí mismo; ello llegó como consecuencia del despliegue de una obra sincera con una búsqueda acorde a un devenir singular, trazado sobre la marcha. Que el proceso haya abierto un nuevo camino, reinterpretando y renovando al tango al mismo tiempo que se nutrió de las mejores tradiciones directamente, es un tipo de experimentación que en vez de negar las herencias más preciadas lo que hizo fue proyectarlas al futuro: "Hay sí una transformación (que nos hace percibir los cambios y el parentesco con el tango anterior), pero también una mutación (que nos muestra la distancia infinita, lo incomparable que tiene Piazzolla con el tango anterior)" (Kuri, 2008: 18). De forma similar, la experimentación etnográfica no constituye necesariamente una negación de la etnografía clásica, sino su consumación o elevación potencial dentro de una dinámica de revitalización constante.

\section{Bibliografía}

Álvarez Pedrosian, E. (2016). Atrevernos a pensarnos a nosotros mismos: un legado foucaultiano. Athenea Digital 16 (1): 291-304. Acceso el 25 de febrero de 2017. Edición electrónica: http:// atheneadigital.net/article/view/v16-n1-alvarez/1522-pdf-es

(2014a). Práctica teórica en emergencia permanente: creación conceptual desde el ejercicio de la etnografía contemporánea. En Cambio conceptual y elección de teorías. Actas del II Coloquio de historia y filosofía de la ciencia, compilado por Pablo Melogno (273299). Montevideo: II-FIC-Udelar.

(2014b). Siglo deleuziano, siglo de los mapas. Cualidad, procesos y sentidos puestos en juego en las cartografías de la subjetividad. Ra'e ga. O espaço geográfico em análise 30: 1140. Edición electrónica: http://revistas.ufpr.br/raega/article/view/36081/22261

. (2011a). Crear, aprender y compartir: apuntes epistemológicos sobre la integralidad. En Integralidad: tensiones y perspectivas (61-83), R. Arocena, H. Tommasino, N. Rodríguez, J. Sutz, E. Álvarez Pedrosian y A. Romano. Montevideo: CSEAM-Udelar.

(2011b). Etnografías de la subjetividad. Herramientas para la investigación. Montevideo: Liccom-Udelar.

(2009). Para no morirse con tantas cosas adentro. Tres operaciones vazferreirianas. Encrucijadas. Diálogos y perspectivas, 1 (3): 185-189. 
(2008). Los efectos de la epistemología kuhneana en las ciencias humanas. En Ciencia, conocimiento y subjetividad (21-38), compilado por Jorge Rasner. Montevideo: CSIC-Udelar.

(2005). Hacer ciencias humanas. Ensayos epistemológicos. Montevideo: Servicio de Publicaciones-FHCE.

(1999). La creatividad como objeto, el montaje como metodología. El cine de Greenaway la antropología contemporánea. En Actas electrónicas de la III RAM. Posadas: Universidad Nacional de Misiones.

Atkinson, P. Delamont, S. y Housley, W. (2007). Contours of culture: complex ethnography and the ethnography of complexity. Lanham (MD): AltaMira Press.

Bachelard, G. (2004) [1938]. La formación del espíritu científico. Contribución a un psicoanálisis del conocimiento objetivo. Siglo XXI: México.

Biehl, J. (2016) [2013]. La etnografía en el camino de la teoría. Etnografías contemporáneas 2 (3): 226-254.

Bourdieu, P. (2003) [2001]. El oficio de científico. Ciencia de la ciencia y reflexividad. Barcelona: Anagrama.

. (1999) [1976]. El campo científico. En Intelectuales, política y poder (75-110). Buenos Aires: Eudeba.

Careri, S. (2009) [2002]. Walkscapes. El andar como práctica estética. Barcelona: Gustavo Gili.

Clifford, J. (1995) [1988]. Sobre el surrealismo etnográfico. En Dilemas de la cultura. Antropología, literatura y arte en la perspectiva postmoderna [The Predicament of Culture] (149-188). Barcelona: Gedisa.

Clifford, J. y Marcus, G. (eds.) (1991) [1986]. Retóricas de la antropología. [Writing Culture: The Poetics and Politics of Ethnography]. Barcelona: Júcar.

Deleuze, G. (1989) [1969]. La lógica del sentido. Barcelona: Paidós.

Deleuze, G. y Guattari, F. (1997a) [1991]. ¿Qué es la filosofía? Barcelona: Anagrama.

(1997b) [1976]. "Rizoma”. En Mil mesetas. Capitalismo y esquizofrenia II (9-32). Valencia: Pre-textos.

Estalella, A. y Sánchez Criado, T. (2016). Experimentación etnográfica: infraestructuras de campo y re-aprendizajes de la antropología. Revista de Dialectología y Tradiciones Populares, LXXI (1): 9-30.

Favret-Saada, J. (2005) [1990]. Ser afetado. Cadernos de campo 14, (13): 155-161.

Feyerabend, P. (1994) [1970]. Contra el método. Esquema de una teoría anarquista del conocimiento. Barcelona: Planeta-Agostini.

Foucault, M. (2002) [1983-1984]. ¿Qué es la Ilustración? Córdoba: Alción Editora.

Geertz, C. (1996) [1973]. La interpretación de las culturas. Barcelona: Gedisa.

. (1989) [1983]. El yo testifical: los hijos de Malinowski. En El antropólogo como autor (83-110). Barcelona: Paidós.

Hastrup, K. y Hervik, P. (edit.) (1994). Social experience and anthropological knowledge. London New York: Routledge.

Ingold, T. (2012). El diseño de ambientes para la vida. En Ambientes para la vida. Conversaciones sobre humanidad, conocimiento y antropología (19-34). Montevideo: CSEAM-Udelar-Trilce.

. (2008). "Anthropology is not ethnography". Proceedings of the British Academy 154: 69-92.

Irwin, K. (2007) [2006]. En el oscuro corazón de la etnografía. Ética y desigualdades en las relaciones íntimas al interior del campo. Apuntes de investigación del CECYP, 12: 133-163. Edición electrónica: http://www.apuntescecyp.com.ar/index.php/apuntes/article/view/279/247

James, W. (2009) [1909]. Un universo pluralista. Filosofía de la experiencia. Buenos Aires: Cactus. Kuri, C. (2008). Agonía del género, potencia del nombre. Constitución de la estética piazzolleana. En Estudios sobre la obra de Astor Piazzolla (13-18), coordinado por Omar García Brunelli. Buenos Aires: Gourmet Musical.

Latour, B. y Woolgar, S. (1995) [1979]. La vida en el laboratorio. La construcción de los hechos científicos. Alianza, Madrid. 
Lins Ribeiro, G. y Escobar, A. (2008) [2006]. Antropologías del mundo. Transformaciones disciplinarias dentro de sistemas de poder. En Antropologías del mundo. Transformaciones disciplinarias dentro de sistemas de poder (11-40), editado por Gustavo Lins Ribeiro y Arturo Escobar. Popayán: The Wenner-Gren Foundation-Envión-CIESAS.

Malinowski, B. (1989) [1967/1914]. Diario de campo en Melanesia. [A diary in the strict sense of the term]. Barcelona: Júcar.

Marcus, G. (2013) [2012]. Los legados de Writing Culture y el futuro cercano de la forma etnográfica: un boceto. Antípoda, 16: 59-80.

. (2008) [2007]. El o los fines de la etnografía: del desorden de lo experimental al desorden de lo barroco. [The end(s) of ethnography: from the messiness of the experimental to the messiness of the baroque]. Revista de Antropología Social 17: 27-48.

Marcus, G. y Fischer, M. (2000) [1986]. La antropología como crítica cultural. Un momento experimental en las ciencias humanas. Buenos Aires: Amorrortu.

Montes-Baquer, J. (2007). El próximo tango. Astor Piazzolla: en diálogo y en concierto. Hamburg: Deutsche Grammophon.

Prigogine, I. y Stengers, I. (1990) [1979]. La nueva alianza. Metamorfosis de la ciencia. Madrid: Alianza.

Rabinow, P. (2009) [2006]. Pasos hacia un laboratorio antropológico. Revista de Antropología Experimental, 9: 137-151.

Reynoso, C. (2015). Crítica de la antropología perspectivista. Viveiros de Castro. Philippe Descola. Bruno Latour. Buenos Aires: Sb.

Reynoso, C. (comp.) (1991). El surgimiento de la antropología posmoderna. México: Gedisa.

Rodríguez Victoriano, J. M. (2004). El oficio de la reflexividad. Notas en torno a Pierre Bourdieu y la tradición cualitativa en la sociología crítica española. En Pierre Bourdieu, las herramientas del sociólogo (299-315), editado por Luis Enrique Alonso, Enrique Martín Criado y José Luis Moreno Pestaña. Madrid: Fundamentos.

Russell, C. (1999). Experimental ethnography. The work of film in the age of video. Durham - London: Duke University Press.

Sáez Rueda, L. (2002). El conflicto entre continentales y analíticos. Dos tradiciones filosóficas. Barcelona: Crítica.

Sansi, R. (2016). Experimentaciones participantes en arte y antropología. Revista de Dialectología y Tradiciones Populares, LXXI (1): 67-73.

Taussig, M. (1995) [1992]. Un gigante en convulsiones. El mundo humano como sistema nervioso en emergencia permanente. [The nervous system]. Barcelona: Gedisa.

Vaz Ferreira, C. (1957) [1938]. Fermentario. Montevideo: Cámara de Representantes de la ROU.

Velasco, H. y Díaz de Rada, Á. (1997). La lógica de la investigación etnográfica. Madrid: Trotta.

Walley, C. (2015). Transmedia as experimental ethnography: The exit Zero Project, deindustrialization, and the politics of nostalgia. American Ethnologist, 42 (4): 624-639. 